\title{
Torsion classes generated by silting modules
}

\author{
Simion Breaz and Jan Žemlička
}

\begin{abstract}
We study the classes of modules which are generated by a silting module. In the case of either hereditary or perfect rings, it is proved that these are exactly the torsion $\mathcal{T}$ such that the regular module has a special $\mathcal{T}$-preenvelope. In particular, every torsion-enveloping class in Mod- $R$ are of the form $\operatorname{Gen}(T)$ for a minimal silting module $T$. For the dual case, we obtain for general rings that the covering torsion-free classes of modules are exactly the classes of the form Cogen $(T)$, where $T$ is a cosilting module.
\end{abstract}

\section{Introduction}

The study of torsion theories which are (co)generated by some special modules is useful since in many cases these torsion theories can be characterized by some intrinsic properties. For instance, it was proved in [1, Proposition 1.1 and Section 2] that in the case of finitely-generated modules over artin algebras the classes of the form gen $(T)$ (i.e. epimorphic images of finite direct sums of copies of $T$ ) induced by a $\tau$-tilting module $T$ coincide with the torsion classes which are enveloping. We refer to [7, Section 5] for similar characterizations in the (co)tilting cases.

The notion of a silting module was introduced in [4] in order to extend the $\tau$-tilting theory, developed in [1] and [15] for finitely generated modules over artin algebras, to infinitely generated modules. The dual notion, i.e. cosilting modules, was studied in [10]. As in the case of tilting modules, see [6], a natural question is to ask for characterizations of torsion classes which are generated by silting modules.

We recall that silting modules are in correspondence with silting objects in the derived category of Mod- $R$, which can be represented by complexes of the form $0 \rightarrow P_{-1} \rightarrow P_{0} \rightarrow 0$ with $P_{-1}$ and $P_{0}$ projective modules. Therefore, they are also in correspondence with important concepts as (co-)t-structures or, in the compact ing.

Key words and phrases: silting, precovering class, preenveloping class, torsion theory, cosilt2010 Mathematics Subject Classification: 16D90, 16E30, 18G15. 
case, with simple-minded collections of objects (see [4] and [16]). It was proved recently that some classes of rings (e.g. hereditary or commutative rings), can be parametrized by universal localizations, [18], Gabriel topologies of finite type, [3], or wide subcategories of finitely presented modules [5]. For other correspondences and constructions, we refer to [19] and [22]. For various correspondences in the cosilting case, we refer to [24] and [25]. Moreover, the 0-th homologies of compact silting complexes of the above form appear naturally as generators for torsion theories $(\mathcal{T}, \mathcal{F})$ in Mod- $R$ such that the heart of the associated $t$-structure is equivalent to a module category, [14] and [17]. For some more general discussions, we refer to [20]. The complexity of the transfer from the finitely-generated case to infinitelygenerated modules is described in [8].

In this paper we provide a general characterization (Proposition 2.1) for silting classes (i.e. classes of the form $\operatorname{Gen}(T)$ for a silting module $T$ ), as torsion classes which are generated via some special pushout constructions. In the case when $R$ is right perfect (Theorem 2.4), respectively right hereditary (Theorem 2.6) it leads to characterizations which can be viewed as extensions of the corresponding result for tilting classes, [6, Theorem 2.1]. In particular, every enveloping torsion class of modules over a perfect ring or over a hereditary ring is generated by a silting module (Corollary 2.12). The case of perfect rings extends the corresponding results proved for finitely-generated modules over an artin algebra in [23] and [1, Theorem 2.7].

The last section of the paper is devoted to the dual setting; namely, we consider torsion-free classes which are of the form Cogen $(T)$, where $T$ is a cosilting module. Since injective modules form an enveloping class over a general ring, we obtain using dual tools that for every ring $R$ torsion-free covering classes in Mod- $R$ are exactly the classes which are cogenerated by cosilting modules (Theorem 3.5).

In this paper $R$ is a unital ring, and Mod- $R$ will denote the category of all right $R$-modules. If $T$ is an $R$-module then $\operatorname{Gen}(T)$ (respectively $\operatorname{Cogen}(T)$ ) denotes the closure to isomorphisms of the class of all quotients (submodules) of direct sums (products) of copies of $T$.

\section{Silting classes}

If $\mathcal{P}$ is the class of all projective modules in $\operatorname{Mod}-R$ and $\mathcal{P} \rightarrow$ will denote the class of all homomorphisms $\sigma: P_{-1} \rightarrow P_{0}$ with $P_{-1}, P_{0} \in \mathcal{P}$.

For every homomorphism $\sigma: P_{-1} \rightarrow P_{0}$ from $\mathcal{P}^{\rightarrow}$ we can associate to $\sigma$ the class

$$
\mathcal{D}_{\sigma}=\left\{X \in \operatorname{Mod}-R \mid \operatorname{Hom}_{R}(\sigma, X) \text { is an epimorphism }\right\} .
$$

If $T$ is a right $R$-module then $\operatorname{Gen}(T)$ denotes the class of all epimorphic images of direct sums of copies of $T$. 
If $\mathcal{T}$ is a class of modules, we will use the following classes:

- ${ }^{\circ} \mathcal{T}=\{X \in \operatorname{Mod}-R \mid \operatorname{Hom}(X, T)=0$ for all $T \in \mathcal{T}\}$,

- $\mathcal{T}^{\circ}=\{X \in \operatorname{Mod}-R \mid \operatorname{Hom}(T, X)=0$ for all $T \in \mathcal{T}\}$,

- ${ }^{\perp} \mathcal{T}=\left\{X \in \operatorname{Mod}-R \mid \operatorname{Ext}^{1}(X, T)=0\right.$ for all $\left.T \in \mathcal{T}\right\}$,

- $\mathcal{T}^{\perp}=\left\{X \in \operatorname{Mod}-R \mid \operatorname{Ext}^{1}(T, X)=0\right.$ for all $\left.T \in \mathcal{T}\right\}$,

- $\square \mathcal{T}=\left\{\alpha \in \mathcal{P} \rightarrow \mid \mathcal{T} \subseteq \mathcal{D}_{\alpha}\right\}$, and

• $\mathcal{T}=\{\operatorname{Coker}(\alpha) \mid \alpha \in \square \mathcal{T}\}$.

Recall from [4] that a module $T$ is partial silting if there exists a projective presentation

$$
P_{-1} \stackrel{\sigma}{\longrightarrow} P_{0} \longrightarrow T \longrightarrow 0
$$

such that $\mathcal{D}_{\sigma}$ is a torsion class and $T \in \mathcal{D}_{\sigma}$. Then $\operatorname{Gen}(T) \subseteq \mathcal{D}_{\sigma} \subseteq T^{\perp}$ and $\left(\operatorname{Gen}(T), T^{\circ}\right)$ is a torsion pair. If $\mathcal{D}_{\sigma}=\operatorname{Gen}(T)$ then $T$ is called a silting module.

Let $\mathcal{T}$ be a class of modules. Then a homomorphism $\varepsilon: X \rightarrow T$ with $T \in \mathcal{T}$ is a $\mathcal{T}$-preenvelope if $\operatorname{Hom}\left(\varepsilon, T^{\prime}\right)$ is surjective for all $T^{\prime} \in \mathcal{T}$, i.e. all homomorphisms $X \rightarrow T^{\prime}$ with $T^{\prime} \in \mathcal{T}$ factorize through $\varepsilon$. The $\mathcal{T}$-preenvelope $\varepsilon$ is a $\mathcal{T}$-envelope if every homomorphism $\alpha: T \rightarrow T$ with the property $\varepsilon=\alpha \varepsilon$ has to be an isomorphism. If all modules $X \in \operatorname{Mod}-R$ have a $\mathcal{T}$-preenvelope (envelope) then $\mathcal{T}$ is preenveloping (resp. enveloping). A preenveloping class $\mathcal{T}$ is special if for every $X \in \operatorname{Mod}-R$ we can find a $\mathcal{T}$-preenvelope $\varepsilon$ which is monic and $\operatorname{Coker}(\varepsilon) \in^{\perp} \mathcal{T}$. The corresponding dual notions are that of (special) precover/precovering and cover/covering, respectively.

Tilting classes, i.e. the torsion classes of the form $\operatorname{Gen}(T)$ with $T$ a tilting module, can be characterized by the fact that they are exactly the special preenveloping torsion classes in Mod- $R$ (cf. [7, Section 5] and [6, Theorem 2.1]). We refer to [11] for a recent study of this kind of special preenveloping situation which involves homomorphisms instead of objects. Even the orthogonality used in this paper does not cover the (co)silting case (cf. [9, Remark 3.2.2]), we can characterize torsion classes of the form $\operatorname{Gen}(T)$ with $T$ a silting module by the existence of preenvelopes with some special properties.

Proposition 2.1. The following are equivalent for a torsion class $\mathcal{T}$ of R-modules:

(1) there exists a silting module $T$ such that $\mathcal{T}=\operatorname{Gen}(T)$;

(2) there exists a $\mathcal{T}$-preenvelope $\varepsilon: R \rightarrow M$ which can be obtained as a pushout

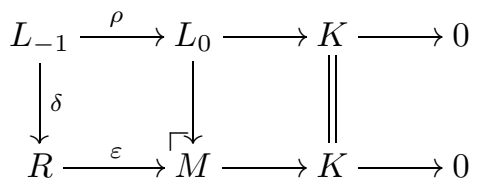

such that $\rho \in \in^{\square} \mathcal{T}$; 
(3) for every $R$-module $X$ there exists an $\mathcal{T}$-preenvelope $\varepsilon: X \rightarrow T_{0}$ which can be obtained as a pushout

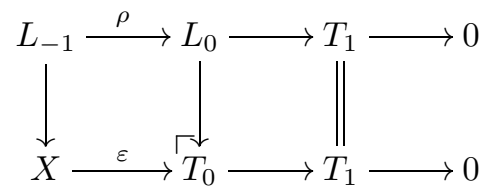

such that $\rho \in \in^{\square} \mathcal{T}$.

If we have a diagram as in (2) then $T=M \oplus K$ is a silting module and $\mathcal{T}=$ $\operatorname{Gen}(T)=\mathcal{D}_{\rho}$.

Proof. (1) $\Rightarrow(3)$ Let $\sigma: P_{-1} \rightarrow P_{0}$ be a homomorphism from $\mathcal{P} \rightarrow$ such that $T=$ $\operatorname{Coker}(\sigma)$, and $T$ is silting with respect to $\sigma$. Hence $\mathcal{T}=\mathcal{D}_{\sigma}$.

For every module $X$ we consider the canonical homomorphism $\delta: P_{-1}^{(I)} \rightarrow X$, where $I=\operatorname{Hom}_{R}\left(P_{-1}, X\right)$, and we construct the pushout diagram

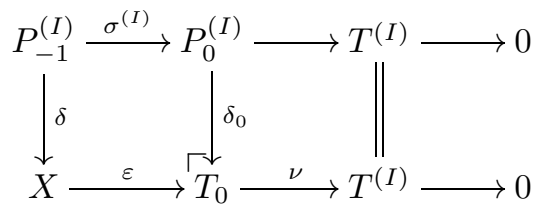

Then, as in the proof of [4, Theorem 3.12] we obtain that $T_{0} \in \mathcal{D}_{\sigma}=\mathcal{T}$.

Moreover, for every $Y \in \mathcal{T}$ and every homomorphism $\alpha: X \rightarrow Y$ there exists $\beta$ : $P_{0}^{(I)} \rightarrow Y$ such that $\delta \alpha=\beta \sigma^{(I)}$. By the pushout universal property there exists $\gamma$ : $T_{0} \rightarrow Y$ such that $\alpha=\gamma \varepsilon$, hence $\varepsilon$ is a $\mathcal{T}$-preenveloping map. Since $\sigma^{(I)} \in \square \mathcal{T}$, the proof is complete.

$(3) \Rightarrow(2)$ is obvious.

$(2) \Rightarrow(1)$ If $X \in \mathcal{D}_{\rho}$ then every homomorphism $R \rightarrow X$ can be lifted to a homomorphism $M \rightarrow X$. Therefore, every element of $X$ is in the image of a homomorphism $M \rightarrow X$, hence $X \in \operatorname{Gen}(M)$. It follows that $\mathcal{D}_{\rho} \subseteq \operatorname{Gen}(M)=\mathcal{T}$. But $\mathcal{T} \subseteq \mathcal{D}_{\rho}$ since $\rho \in \square \mathcal{T}$, and it follows that $\mathcal{D}_{\rho}=\mathcal{T}$ is a torsion class. Moreover, $K \in \mathcal{T}=\mathcal{D}_{\rho}$, hence $K$ is partial silting with respect to $\rho$. By the proof of [4, Theorem 3.12] it follows that $T=M \oplus K$ is a silting module with respect to the projective resolution

$$
\gamma \oplus \rho: L_{-1} \oplus L_{-1} \longrightarrow\left(L_{0} \oplus R\right) \oplus L_{0},
$$

where $L_{-1} \stackrel{\gamma}{\rightarrow} L_{0} \oplus R \rightarrow M$ is the canonical exact sequence induced by $\delta$ and $\rho$, and that $\operatorname{Gen}(T)=\mathcal{D}_{\rho}=\mathcal{T}$.

In order to apply the above proposition we will use the following characterization for pushout diagrams. 
Lemma 2.2. In a commutative diagram

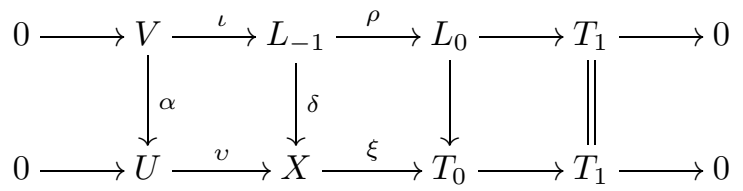

the middle square is a pushout if and only if $\alpha$ is an epimorphism.

Proof. Suppose that the middle square is a pushout. We consider $\pi: U \rightarrow$ $U / \operatorname{Im}(\alpha)$ the canonical epimorphism, and $\mu: U / \operatorname{Im}(\alpha) \rightarrow E$ is the embedding of $U / \operatorname{Im}(\alpha)$ into its injective envelope. There exists a homomorphism $\nu: X \rightarrow E$ such that $\nu v=\mu \pi$, hence $\nu \delta \iota=0$. Then $\nu \delta$ factorizes through $\operatorname{Coker}(\iota)$. Since $E$ is injective and the top horizontal line is an exact sequence, it follows that $\nu \delta$ factorizes through $\rho$. Moreover, the middle square is a pushout, and we obtain that $\nu$ factorizes through $\xi$. It follows that $\mu \pi=\nu v=0$. Since $\mu$ is monic, we obtain $\pi=0$, hence $\alpha$ is an epimorphism.

Conversely, if $\alpha$ is an epimorphism and we have two homomorphisms $\beta_{1}: X \rightarrow Y$ and $\beta_{2}: L_{0} \rightarrow Y$ such that $\beta_{1} \delta=\beta_{2} \rho$ then $\beta_{1} v \alpha=0$, hence $\beta_{1} v=0$. It follows that there exists a unique homomorphism $\bar{\beta}: \operatorname{Im}(\xi) \rightarrow Y$ such that $\beta_{1}=\bar{\beta} \bar{\xi}$, where $\bar{\xi}: X \rightarrow \operatorname{Im}(\xi)$ is the homomorphism induced by $\xi$.

Let $\bar{\delta}: \operatorname{Im}(\rho) \rightarrow \operatorname{Im}(\xi)$ be the homomorphism induced by $\delta$. If $\iota_{\rho}: \operatorname{Im}(\rho) \rightarrow L_{0}$ and $\iota_{\xi}: \operatorname{Im}(\xi) \rightarrow T_{0}$ are the canonical inclusions, then $\bar{\beta} \bar{\delta}=\beta_{2} \iota_{\rho}$. Since the first square in the commutative diagram

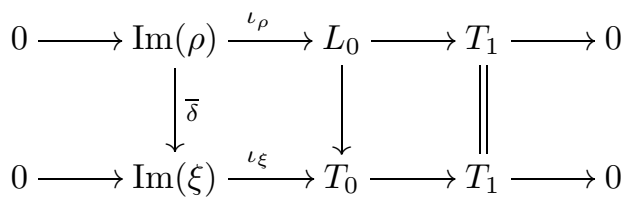

is a pushout, there exists a unique homomorphism $\beta^{*}: T_{0} \rightarrow Y$ such that $\bar{\beta}$ (hence $\beta_{1}$ ) and $\beta_{2}$ factorize through $\beta^{*}$, and the proof is complete.

Let $Y$ be a submodule of a module $P$, and consider a canonical projection $\pi: P \rightarrow P / Y$. Recall that $Y \ll P$ means that $Y$ is a superfluous submodule of a module $P$, i.e. that $\varphi$ is an epimorphism for every $\varphi \in \operatorname{Hom}(M, P)$ such that $\pi \varphi$ is an epimorphism.

We will need the following easy observation:

Lemma 2.3. Let $X, P, T$ be modules over a ring $R$ such that $X \ll P$ and $\alpha \in$ $\operatorname{Hom}(P, T)$. Then $\alpha(X) \ll \alpha(P)$. If, furthermore, $\alpha(X)=\alpha(P)$ then $\alpha=0$. 
Now we are ready to characterize torsion classes generated by silting modules over right perfect rings.

Theorem 2.4. Let $R$ be a right perfect ring and $\mathcal{T} \subseteq$ Mod- $R$ a torsion class. The following are equivalent:

(1) $\mathcal{T}=\operatorname{Gen}(T)$ for a silting module $T$;

(2) There exists a $\mathcal{T}$-preenvelope $\varepsilon: R \rightarrow M$ such that $M \in \mathcal{T} \cap{ }^{\perp} \mathcal{T}$.

In these conditions, if $K=\operatorname{Coker}(\varepsilon)$ then $M \oplus K$ is a silting module, and $\mathcal{T}=$ $\operatorname{Gen}(M \oplus K)$.

Proof. $(1) \Rightarrow(2)$ This is a consequence of Proposition 2.1 (see also [4, Proposition 3.11]).

$(2) \Rightarrow(1)$ We consider the exact sequence $0 \rightarrow U \stackrel{\iota}{\rightarrow} R \stackrel{\varepsilon}{\rightarrow} M \stackrel{\rho}{\rightarrow} K \rightarrow 0$ where $U=$ $\operatorname{Ker}(\varepsilon)$ and $\iota_{U}$ is the inclusion map, and we will construct a commutative diagram

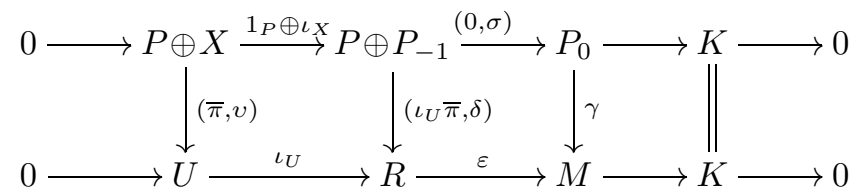

such that its horizontal lines are exact sequences, and

(1) $P, P_{-1}$, and $P_{0}$ are projective modules;

(2) $(0, \sigma) \in^{\square} \mathcal{T}$;

(3) $(\bar{\pi}, v)$ is surjective.

If such a diagram is constructed then it remains to apply Lemma 2.2 and Proposition 2.1 to conclude that $\mathcal{T}$ is generated by a silting module.

Step 1. We will construct a commutative diagram

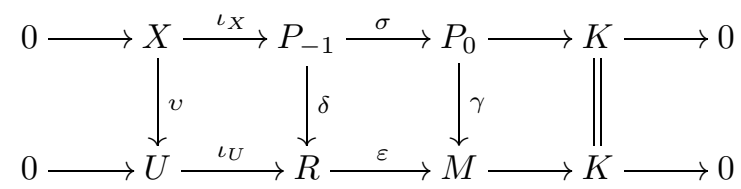

such that the horizontal lines are exact sequences, $P_{-1}$ and $P_{0}$ are projective modules, and $X=\operatorname{Ker}(\sigma)$ is superfluous in $P_{-1}$.

If $\bar{\varepsilon}: R / U \rightarrow M$ is the homomorphism induced by $\varepsilon$ then for every $T \in \mathcal{T}$ the homomorphism $\operatorname{Hom}(\bar{\varepsilon}, T)$ is an epimorphism. Since $M \in^{\perp} \mathcal{T}$ we obtain $K \in^{\perp} \mathcal{T}$. 
For an epimorphism $\gamma: P_{0} \rightarrow M$ with $P_{0}$ projective, we have a commutative diagram whose horizontal lines are exact sequences

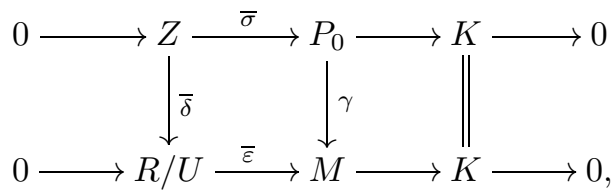

where $\bar{\sigma}$ and $\bar{\varepsilon}$ are the canonical homomorphisms induced by $\sigma$ and $\varepsilon$, respectively. Since $R$ is right perfect, we can take a projective cover $\pi_{\sigma}: P_{-1} \rightarrow Z$ of $Z$, and we use it to complete the above diagram to the commutative diagram

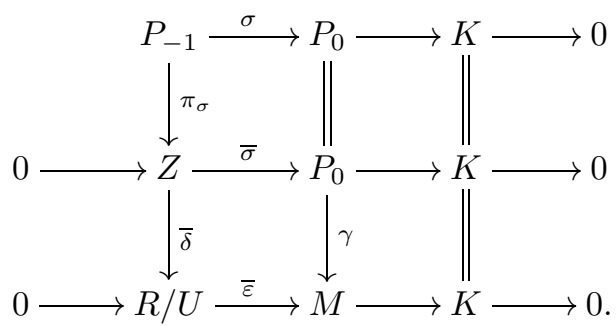

Note that $\pi_{\sigma}$ is onto, so the horizontal lines in this diagram are exact sequences.

Since $P_{-1}$ is projective we can construct a commutative diagram (2) such that the horizontal lines are exact sequences, and $\pi_{U} \delta=\bar{\delta} \pi_{\sigma}$, where $\pi_{U}: R \rightarrow R / U$ is the canonical projection and $\iota_{X}$ is the inclusion map. Moreover, since $X=\operatorname{ker} \sigma=\operatorname{ker} \pi_{\sigma}$, we observe that $X \ll P_{-1}$.

Claim 1. For every $T \in \mathcal{T}$ the homomorphism $\operatorname{Hom}(\sigma, T)$ is onto.

In order to prove this claim we will use techniques which are similar to those used in [8]. Let us consider the short exact sequence

$$
0 \longrightarrow Z \stackrel{\bar{\sigma}}{\longrightarrow} P_{0} \longrightarrow K \longrightarrow 0
$$

and note that for every $T \in \mathcal{T}$ we have a short exact sequence

$$
0 \longrightarrow \operatorname{Hom}(K, T) \longrightarrow \operatorname{Hom}\left(P_{0}, T\right) \stackrel{\operatorname{Hom}(\bar{\sigma}, T)}{\longrightarrow} \operatorname{Hom}(Z, T) \longrightarrow 0
$$

since $\operatorname{Ext}^{1}(K, T)=0$. Fix an arbitrary $T \in \mathcal{T}$ and an arbitrary $\varphi \in \operatorname{Hom}\left(P_{-1}, T\right)$. Let us denote by $\pi_{X}: P_{-1} \rightarrow P_{-1} / X$ and $\pi_{T}: T \rightarrow T / \varphi(X)$ the canonical projections. Then we can find a homomorphism $\bar{\varphi} \in \operatorname{Hom}\left(P_{-1} / X, T / \varphi(X)\right)$ which satisfies $\bar{\varphi} \pi_{X}=$ $\pi_{T} \varphi$. As $T / \varphi(X) \in \mathcal{T}$, there exists $\bar{\psi} \in \operatorname{Hom}\left(P_{0}, T / \varphi(X)\right)$ for which $\bar{\psi} \sigma=\bar{\varphi} \pi_{X}$ by the 
exactness of (3). Since $P_{0}$ is projective and $\pi_{T}$ is an epimorphism, $\bar{\psi}$ factorizes through $\pi_{T}$, i.e. there exists $\psi \in \operatorname{Hom}\left(P_{0}, T\right)$ such that $\pi_{T} \psi=\bar{\psi}$. Hence

$$
\pi_{T} \psi \sigma=\bar{\psi} \sigma=\bar{\varphi} \pi_{X}=\pi_{T} \varphi
$$

Put $\alpha:=\varphi-\psi \sigma \in \operatorname{Hom}\left(P_{-1}, T\right)$. From $\pi_{T} \alpha=0$ we have $\alpha\left(P_{-1}\right) \subseteq \varphi(X)$. Furthermore, $\left.\alpha\right|_{X}=\left.\varphi\right|_{X}$ since $\psi \sigma(X)=0$, which implies that $\alpha\left(P_{-1}\right) \subseteq \alpha(X)$. By Lemma 2.3 we obtain $\alpha=0$, so $T \in \mathcal{D}_{\sigma}$, and the claim is proved.

Since $v$ is not necessarily surjective, we have to modify diagram (2). In order to do this we will pass to the second step of the proof:

Step 2. We will study the properties of the homomorphisms involved in diagram (2).

Using the pushout of $\sigma$ and $\delta$ we obtain a commutative diagram

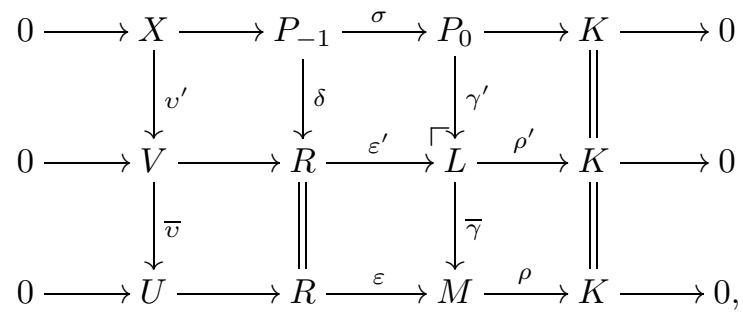

such that the horizontal lines are exact sequences, $\bar{v} v^{\prime}=v$, and $\gamma=\bar{\gamma} \gamma^{\prime}$. In order to simplify the presentation, let us remark that $v^{\prime}$ is surjective (by Lemma 2.2) and $\bar{v}$ is injective, hence $V$ can be identified with the image of $v$. In this case the equality $\bar{v} v^{\prime}=v$ represents the canonical decomposition of $v$ through its image.

Claim 2. $\operatorname{Hom}(\operatorname{Ker}(\bar{\gamma}), \mathcal{T})=0$.

In order to prove this we will prove that $\bar{\gamma}$ is a $\mathcal{T}$-preenvelope for $L$. For every $T \in \mathcal{T}$ and every homomorphism $\alpha: L \rightarrow T$ there exists $\bar{\alpha}: M \rightarrow T$ such that $\alpha \varepsilon^{\prime}=\bar{\alpha} \varepsilon$. Then $(\bar{\alpha} \bar{\gamma}-\alpha) \varepsilon^{\prime}=0$, hence there exists $\beta: K \rightarrow T$ such that $\bar{\alpha} \bar{\gamma}-\alpha=\beta \rho^{\prime}=\beta \rho \bar{\gamma}$. Then $\alpha=(\bar{\alpha}-\beta \rho) \bar{\gamma}$, hence $\bar{\gamma}$ is a $\mathcal{T}$-preenvelope for $L$.

Therefore, since $M \in^{\perp} \mathcal{T}$, applying the functors $\operatorname{Hom}(-, T)$ with $T \in \mathcal{T}$ to the exact sequence $0 \rightarrow \operatorname{Ker}(\bar{\gamma}) \rightarrow L \rightarrow M \rightarrow 0$ it follows that $\operatorname{Hom}(\operatorname{Ker}(\bar{\gamma}), T)=0$ for all $T \in \mathcal{T}$, and the claim is proved.

Claim 3. $\operatorname{Coker}(\bar{v}) \cong \operatorname{Ker}(\bar{\gamma})$.

In order to prove this, we split the bottom rectangle in the diagram (4) in two commutative diagrams with short exact sequences, 


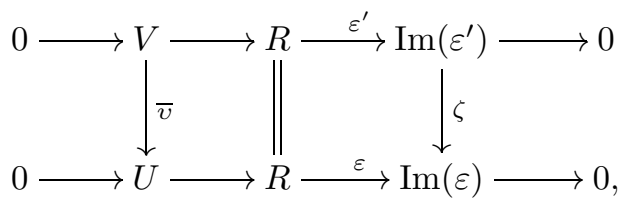

and

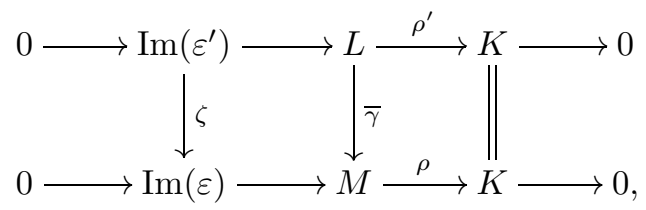

where $\zeta$ can be identified to the canonical surjection $R / V \rightarrow R / U$. Applying KerCoker Lemma, we observe that $\operatorname{Coker}(\bar{v}) \cong \operatorname{Ker}(\bar{\gamma})$.

Step 3. The construction of diagram (1).

Since $R$ is right perfect, there exists $\pi: P \rightarrow \operatorname{Coker}(\bar{v})$ is a projective cover for $\operatorname{Coker}(\bar{v})$. Moreover, $\operatorname{Coker}(\bar{v})$ is an epimorphic image of $U$, hence we can lift $\pi$ to a homomorphism $\bar{\pi}: P \rightarrow U$. Now it is easy to see that we have obtained the commutative diagram (1).

Claim 4. $(0, \sigma) \in \square \mathcal{T}$.

Let $\alpha: P \rightarrow T$ be a homomorphism with $T \in \mathcal{T}$. It induces a homomorphism $\bar{\alpha}: P / \operatorname{Ker}(\pi) \rightarrow T / \alpha(\operatorname{Ker}(\pi))$ defined by the rule

$$
\bar{\alpha}(x+\operatorname{Ker}(\pi))=\alpha(x)+\alpha(\operatorname{Ker}(\pi)) .
$$

Using the isomorphisms $P / \operatorname{Ker}(\pi) \cong \operatorname{Coker}(\bar{v}) \cong \operatorname{Ker}(\bar{\gamma})$ and Claim 2, it follows that $\bar{\alpha}=0$. Therefore $\alpha(P)=\alpha(\operatorname{Ker}(\pi))$. Since $\operatorname{Ker}(\pi)$ is superfluous, it follows that $\alpha=0$, hence $\operatorname{Hom}(P, \mathcal{T})=0$. Using Claim 1 , we obtain that $\mathcal{T} \subseteq \mathcal{D}_{(0, \sigma)}$ and the proof of the claim is complete.

Therefore, in order to complete the proof, it is enough to prove

Claim 5. $(\bar{\pi}, v)$ is an epimorphism.

By Lemma 2.2, $v^{\prime}$ is an epimorphism, and it is easy to see that $\operatorname{Im}(v)+\operatorname{Im}(\bar{\pi})=$ $\operatorname{Im}(\bar{v})+\operatorname{Im}(\bar{\pi})=U$, and the claim is proved.

The following class of examples, used in commutative case also in [3, Example 5.4 ], shows that the implication $(2) \Rightarrow(1)$ does not hold in general. For the general theory of semiperfect rings we refer to [2]. 
Example 2.5. Let $R$ be a semiperfect ring with non-zero idempotent Jacobson radical $J$, i.e. $J^{2}=J \neq 0$. An example of such a ring is constructed in $[21$, Exercise 10.2]. Denote by $S_{i}$ the simple modules and by $P_{i}$ the corresponding indecomposable projectives such that $\oplus_{i \leq n} S_{i}=R / J$ and $S_{i} \cong P_{i} / P_{i} J[2$, Proposition 27.10 and Theorem 27.11]. Since idempotency of $J$ implies that extensions of semisimple modules by semisimple modules are semisimple as well, we can see that $\mathcal{T}=\operatorname{Gen}(R / J)=\left\{\oplus_{i} S_{i}^{\left(\varkappa_{i}\right)} \mid \varkappa_{i}, i \leq n\right\}$ is a torsion class and $\operatorname{Ext}^{1}(T, U)=0$ for each $T, U \in \mathcal{T}$, hence $R / J \in \mathcal{T} \cap^{\perp} \mathcal{T}$. Furthermore, it is easy to verify that the natural projection $R \rightarrow R / J$ forms a $\mathcal{T}$-envelope of $R$. We will show that no generator $G=\oplus_{i} S_{i}^{\left(\varkappa_{i}\right)}$ of $\mathcal{T}$ is silting.

Consider an exact sequence $P_{-1} \stackrel{\sigma}{\rightarrow} P_{0} \stackrel{\rho}{\rightarrow} \oplus_{i} S_{i}^{\left(\varkappa_{i}\right)} \rightarrow 0$. Since $\rho$ factorizes through the canonical projection $\pi: \oplus_{i} P_{i}^{\left(\varkappa_{i}\right)} \rightarrow \oplus_{i} S_{i}^{\left(\varkappa_{i}\right)}$ we may suppose that $P_{0}=\oplus_{i} P_{i}^{\left(\varkappa_{i}\right)}$ and that $\rho=\pi$. Note that $\operatorname{Im}(\sigma)=\oplus_{i} P_{i}^{\left(\varkappa_{i}\right)} J \neq 0$ because $\oplus_{i} S_{i}^{\left(\varkappa_{i}\right)}$ generates $\mathcal{T}$, which implies that $P_{-1} \neq 0$. Since for every $T \in \mathcal{T}$ and every homomorphism $\varphi \in \operatorname{Hom}\left(P_{0}, T\right)$ we have $\operatorname{Im}(\sigma)=P_{0} J \subseteq \operatorname{ker}(\varphi)$, the composition $\varphi \sigma$ is zero. As $\operatorname{Hom}(\sigma, T)=0$ while $\operatorname{Hom}\left(P_{-1}, T\right) \neq 0$ for all nonzero $T \in \mathcal{T}$, we can conclude that $G$ is not silting.

Finally note that the class of semiperfect rings with non-zero idempotent Jacobson radical contains for example all valuation domains with infinitely generated maximal ideals.

A similar result is valid for hereditary (which are not necessarily right perfect) rings. In this case silting torsion classes can be characterized by the existence of a special long exact sequence. We note that the equivalence of (1) and (2) in the next result can be deduced from [5, Lemma 5.1 and Proposition 5.2] by using the characterization of tilting classes as special preenveloping torsion classes, $[6$, Theorem 2.1].

Theorem 2.6. Let $R$ be a right hereditary ring and $\mathcal{T} \subseteq$ Mod- $R$ a torsion class. The following are equivalent:

(1) $\mathcal{T}=\operatorname{Gen}(T)$ for a silting module $T$;

(2) there exists a $\mathcal{T}$-preenvelope $\varepsilon: R \rightarrow M$ such that $M \in \mathcal{T} \cap \perp \mathcal{T}$;

(3) there exists an exact sequence $0 \rightarrow U \rightarrow R \rightarrow M \rightarrow K \rightarrow 0$ such that $M \in \mathcal{T}, U \in$ ${ }^{\circ} \mathcal{T}$ and $K \in{ }^{\perp} \mathcal{T}$.

Proof. $(1) \Rightarrow(2)$ The argument is the same as in the proof of Theorem 2.4, i.e. we apply Proposition 2.1.

$(2) \Rightarrow(3)$ As in the proof of Theorem 2.4 we obtain $K \in^{\perp} \mathcal{T}$.

Since $\varepsilon$ is a $\mathcal{T}$-preenvelope, every homomorphism $R \rightarrow T$ with $T \in \mathcal{T}$ factorizes through $R / U$. Therefore, for every $T \in \mathcal{T}$ we have that $\operatorname{Hom}(\pi, T)$ is an isomorphism, 
where $\pi: R \rightarrow R / U$ is the canonical epimorphism. Then first natural homomorphism from the exact sequence

$$
0 \longrightarrow \operatorname{Hom}(R / U, T) \longrightarrow \operatorname{Hom}(R, T) \longrightarrow \operatorname{Hom}(U, T) \longrightarrow \operatorname{Ext}^{1}(R / U, T)
$$

is an isomorphism. Moreover, using the exact sequence $0 \rightarrow R / U \rightarrow M \rightarrow K \rightarrow 0$, we obtain $\operatorname{Ext}^{1}(R / U, T)=0$ for all $T \in \mathcal{T}$. Therefore $\operatorname{Hom}(U, T)=0$ for all $T \in \mathcal{T}$.

$(3) \Rightarrow(1)$ Since $R$ is hereditary, there exists a projective resolution

$$
0 \longrightarrow P_{-1} \stackrel{\sigma}{\longrightarrow} P_{0} \longrightarrow K \longrightarrow 0
$$

Using the hypothesis $K \in^{\perp} \mathcal{T}$, it follows that $\sigma \in^{\square} \mathcal{T}$. If $U=\operatorname{Ker}(\varepsilon)$ we can construct, as in the proof of Theorem 2.4, using the projectivity of $P_{0}$, a commutative diagram

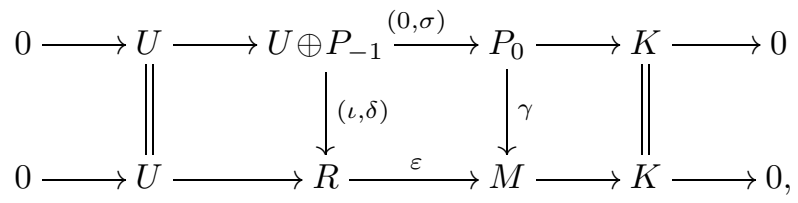

where $\iota: U \rightarrow R$ is the inclusion map. Since $U$ is projective, by $U \in^{\circ} \mathcal{T}$ it follows that $(0, \sigma) \in^{\square} \mathcal{T}$. From Proposition 2.1 we conclude that $\operatorname{Gen}(T)=\mathcal{T}$ is a silting class.

The following example shows that the condition $U \in^{\circ} \mathcal{T}$ is essential in the proof of $(3) \Rightarrow(1)$. We refer to [12] for the general theory of injective abelian groups.

Example 2.7. Let $\mathcal{T}=\operatorname{Gen}\left(\mathbb{Z}\left(p^{\infty}\right)\right)$ in the category Mod- $\mathbb{Z}$ for a prime number $p$, where $\mathbb{Z}\left(p^{\infty}\right)$ is the $p$-component of the abelian group $\mathbb{Q} / \mathbb{Z}$. It is easy to see that $\mathcal{T}$ is the class of all injective abelian $p$-groups, so it is a torsion class and for every $K \in \mathcal{T}$ we have $K \in^{\perp} \mathcal{T}$. Therefore, for every homomorphism $\varepsilon: \mathbb{Z} \rightarrow M$ with $M \in \mathcal{T}$ we have $\operatorname{Coker}(\varepsilon) \in^{\perp} \mathcal{T}$ and $\operatorname{Ker}(\varepsilon) \cong \mathbb{Z} \notin^{\circ} \mathcal{T}$. By [4, Corollary 3.5 and Proposition 3.10] every torsion class generated by a silting module is closed under direct products. Since $\mathbb{Z}\left(p^{\infty}\right)^{\aleph_{0}}$ is not a torsion group, it follows that $\mathcal{T}$ is not closed under direct products, hence $\mathcal{T}$ is not generated by a silting module.

On the other side, in the case of perfect rings there exists a torsion class $\mathcal{T}$ generated by a silting module such that $U \notin^{\circ} \mathcal{T}$.

Example 2.8. We consider, as in [4, Example 4.1] the $k$-algebra

$$
R=k Q /(\alpha \beta \alpha, \beta \alpha \beta),
$$


where $Q$ is the quiver

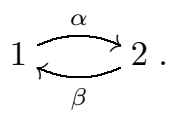

If $S_{1}$ and $S_{2}$ are the simple $R$-modules, respectively $P_{1}$ and $P_{2}$ are the corresponding projectives, then $M=S_{1} \oplus P_{1} \oplus P_{1}$ is a silting module. A Gen $(M)$-preenvelope for $R$ is given by

$$
0 \longrightarrow U \longrightarrow P_{1} \oplus P_{2} \stackrel{1_{P_{1}} \oplus \varphi}{\longrightarrow} P_{1} \oplus P_{1} \longrightarrow S_{1} \longrightarrow 0
$$

where $P_{2} \stackrel{\varphi}{\rightarrow} P_{1} \rightarrow S_{1} \rightarrow 0$ is the minimal projective presentation for $S_{1}$. It is not hard to see that $\operatorname{Hom}\left(U, S_{1}\right) \cong \operatorname{Ext}^{1}\left(S_{2}, S_{1}\right) \neq 0$.

Let us recall that a module $T$ (not necessarily finitely generated) is tilting if $\operatorname{Gen}(T)=T^{\perp}$. The module $T$ is quasi-tilting if $\operatorname{Pres}(T)=\operatorname{Gen}(T) \subseteq T^{\perp}$, and $T$ is finendo if it is finitely generated as a left module over its endomorphism ring. By [4, Proposition 3.10], the class of silting modules is an intermediate class between the class of tilting modules and that of finendo quasi-tilting modules. Using a theorem of Wei, [26], it is proved in [4, Proposition 3.15] that in the case of finitely generated modules over finitely dimensional algebras the silting finitely generated modules coincide to (finendo) quasi-tilting modules. In the case of hereditary or right perfect rings, we obtain a similar result. We note that the hereditary case in the following corollary was also proved in [5, Proposition 5.2 and Example 5.5].

Corollary 2.9. Let $R$ be a right hereditary or right perfect ring. If $Q$ is a finendo quasi-tilting module then there exists a silting module $T$ such that $\operatorname{Add}(Q)=$ $\operatorname{Add}(T)$.

Consequently, the following are equivalent for a torsion class $\mathcal{T} \subseteq$ Mod-R:

(1) $\mathcal{T}=\operatorname{Gen}(T)$ for a silting module $T$;

(2) $\mathcal{T}=\operatorname{Gen}(T)$ for a finendo quasi-tilting module $T$.

Proof. Let us recall from [4, Proposition 3.2 and Theorem 3.4] that $Q$ is finendo quasi-tilting if and only if there exists an exact sequence

$$
R \stackrel{\alpha}{\longrightarrow} Q_{0} \longrightarrow Q_{1} \longrightarrow 0
$$

such that $\alpha$ is a $\operatorname{Gen}(Q)$-preenvelope, $Q_{0}, Q_{1} \in \operatorname{Add}(Q)$ and $Q_{1} \in{ }^{\perp} \operatorname{Gen}(Q)$. From the proof of Theorem 2.4 and Theorem 2.6 it follows that $T=Q_{0} \oplus Q_{1}$ is a silting module. Not it is easy to see that $\operatorname{Add}(Q)=\operatorname{Add}(T)$ and $\operatorname{Gen}(Q)=\operatorname{Gen}(T)$.

Using [3, Example 5.4] we observe that the equivalence from the above corollary is not true for general rings. 
Example 2.10. Let $R$ be a commutative local ring such that its maximal ideal is idempotent. Then the simple module $S$ is $\operatorname{Mod}-R$ is finendo quasi-tilting. But, we proved in Example 2.5 that $\operatorname{Gen}(S)$ is not generated by a silting module.

We recall from [5] that a silting module $T$ is minimal if there exists a Gen $(T)$-envelope for the regular module $R$. In order to apply the above results to minimal silting modules we need a lemma whose proof is included for reader's convenience.

Lemma 2.11. Let $\mathcal{T}$ be a class of modules. If $\varepsilon: R \rightarrow M$ is an $\mathcal{T}$-envelope then every epimorphism $\alpha: N \rightarrow M$ with $N \in \mathcal{T}$ splits. Consequently, if $\mathcal{T}$ is a class closed under extensions and $\varepsilon: R \rightarrow M$ is a $\mathcal{T}$-envelope then $M \in^{\perp} \mathcal{T}$.

Proof. Since $\alpha$ is an epimorphism, there exists $\gamma: R \rightarrow N$ such that $\varepsilon=\alpha \gamma$. Then there exists $\beta: M \rightarrow N$ such that $\beta \varepsilon=\gamma$. It follows that $\alpha \beta \varepsilon=\varepsilon$. Since $\varepsilon$ is a $\mathcal{T}$-envelope, it follows that $\alpha \beta$ is an automorphism, hence $\alpha$ splits.

The last statement is now obvious since in every short exact sequence $0 \rightarrow T \rightarrow$ $N \rightarrow M \rightarrow 0$, with $T \in \mathcal{T}$, we have $N \in \mathcal{T}$.

Corollary 2.12. The following are equivalent for a torsion class $\mathcal{T}$ of modules over a right hereditary or right perfect ring $R$ :

(1) $\mathcal{T}=\operatorname{Gen}(T)$ for a minimal silting module $T$;

(2) There exists a $\mathcal{T}$-envelope $\varepsilon: R \rightarrow M$.

In particular, all enveloping torsion classes over hereditary or right perfect rings are generated by silting modules.

Moreover, a half of Salce's Lemma [13, Lemma 5.20] is valid for silting modules:

Proposition 2.13. Let $T$ be a silting module. If $\mathcal{T}=\operatorname{Gen}(T)$ then for every $R$-module $X$ there exists a short exact sequence

$$
0 \longrightarrow L \longrightarrow U \stackrel{v}{\longrightarrow} X \longrightarrow 0
$$

such that $v$ is $a \diamond \mathcal{T}$-precover for $X$ and $L \in \mathcal{T}$.

Consequently, ${ }^{\perp} \mathcal{T}$ is a special precovering class.

Proof. If $X$ is an $R$-module, we consider a pushout diagram

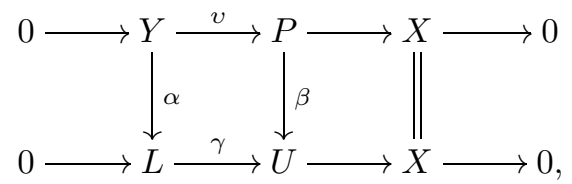

where $P$ is a projective module, $L \in \mathcal{T}$, and $\alpha: Y \rightarrow L$ is a $\mathcal{T}$-preenvelope for $Y$ obtained as a pushout 


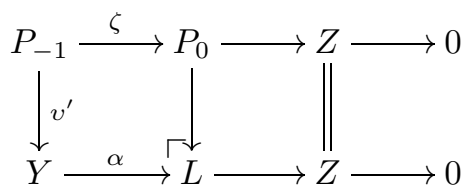

for some $\zeta \in^{\square} \mathcal{T}$. Then we have a pushout square

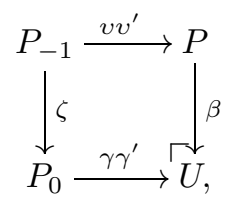

hence $U$ is the cokernel of the homomorphism $\delta: P_{-1} \rightarrow P_{0} \oplus P$ induced by $v v^{\prime}$ and $\zeta$. Since every homomorphism $f: P_{-1} \rightarrow T$ with $T \in \mathcal{T}$ can be written as $f=g \zeta$ for some $g: P_{0} \rightarrow T$, it follows that $f=g^{\prime} \delta$, where $g^{\prime}: P_{0} \oplus P \rightarrow T$ is defined by $g_{\mid P_{0}}^{\prime}=g$ and $g_{\mid P}^{\prime}=0$. Then $\delta \in^{\square} \mathcal{T}$, so $U \in^{\diamond} \mathcal{T}$.

Now, for every $V \in^{\diamond} \mathcal{T}$ we have $\mathcal{T} \subseteq V^{\perp}$, and it follows that $\gamma$ is a ${ }^{\diamond} \mathcal{T}$-precover for $X$.

The last statement follows from the inclusion ${ }^{\diamond} \mathcal{T} \subseteq{ }^{\perp} \mathcal{T}$.

\section{Cosilting classes}

For the dual results, let us recall from [10] that we can associate to every homomorphism $\sigma: Q_{0} \rightarrow Q_{1}$ between injective modules the class

$$
\mathcal{B}_{\sigma}=\left\{X \in \operatorname{Mod}-R \mid \operatorname{Hom}_{R}(X, \sigma) \text { is an epimorphism }\right\},
$$

and a module $T$ is partial cosilting if there exists an injective presentation

$$
0 \longrightarrow T \longrightarrow Q_{0} \stackrel{\sigma}{\longrightarrow} Q_{1}
$$

such that $\mathcal{B}_{\sigma}$ is a torsion-free class and $T \in \mathcal{B}_{\sigma}$. Then $\operatorname{Cogen}(T) \subseteq \mathcal{B}_{\sigma} \subseteq{ }^{\perp} T$. If $\mathcal{B}_{\sigma}=$ Cogen $(T)$ then $T$ is called cosilting.

Let $\mathcal{I}$ be the class of all injective modules, and $\mathcal{I}^{\rightarrow}$ the class of all homomorphisms between injective modules. If $\mathcal{F}$ is a class of right $R$-modules then we associate to $\mathcal{F}$ the following classes

- $\mathcal{F}^{\square}=\left\{\sigma: S_{0} \rightarrow S_{1} \mid \sigma \in \mathcal{I}^{\rightarrow}\right.$, and $\left.\mathcal{F} \subseteq \mathcal{B}_{\sigma}\right\}$, and

- $\mathcal{F}^{\diamond}=\left\{\operatorname{Ker}(\sigma) \mid \sigma \in \mathcal{F}^{\square}\right\}$.

In order to dualize Theorem 2.4 and Corollary 2.12 let us formulate dual versions of Propositions 2.1. 
Proposition 3.1. The following are equivalent for a torsion-free class $\mathcal{F}$ of R-modules:

(1) There exists a cosilting module $T$ such that $\mathcal{F}=\operatorname{Cogen}(T)$;

(2) If $E$ is a fixed injective cogenerator for Mod- $R$ then there exists an $\mathcal{F}$-precover $\varepsilon: M \rightarrow E$ which can be obtained as a pullback

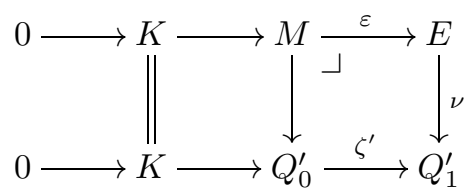

such that $\zeta^{\prime} \in \mathcal{F}^{\square}$;

(3) For every $R$-module $X$ there exists an $\mathcal{F}$-precover $\alpha: M \rightarrow X$ which can be obtained as a pullback

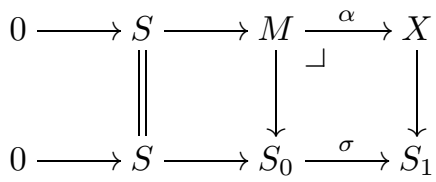

such that $\sigma \in \mathcal{F}^{\square}$.

If we have a diagram as in (2) then $K \oplus M$ is a cosilting module and $\mathcal{F}=$ $\operatorname{Cogen}(K \oplus M)$.

If $Y$ is a submodule of a module $P$ with the canonical embedding $\nu: Y \rightarrow P$, then $Y$ is an essential submodule of $P, Y \unlhd P$, if an arbitrary homomorphism $\varphi \in$ $\operatorname{Hom}(P, N)$ is a monomorphism whenever $\varphi \nu$ is a monomorphism.

Lemma 3.2. Let $Y, Q, F$ be modules over a ring $R$ such that $Y \unlhd Q$ and $\alpha \in$ $\operatorname{Hom}(F, Q)$. Then $\beta(F) \cap Y \unlhd \beta(F)$. If, furthermore, $\beta(F) \cap Y=0$, then $\beta=0$.

We will also use the dual of Lemma 2.11.

Lemma 3.3. Suppose that $\mathcal{F}$ is a class of modules and $\varepsilon: M \rightarrow E$ is an $\mathcal{F}$-cover of an injective module $E$. Then every monomorphism $\alpha: M \rightarrow N$ with $N \in \mathcal{F}$ splits.

Therefore, if $\mathcal{F}$ is a class closed under extensions and $\varepsilon: M \rightarrow E$ is an $\mathcal{F}$-cover of an injective module $E$ then $M \in \mathcal{F}^{\perp}$.

As in the (co)tilting theory, we obtain the following:

Lemma 3.4. If $T$ is a cosilting module then $\operatorname{Cogen}(T)$ is a covering class.

Proof. It is proved in [10, Corollary 4.8] that Cogen $(T)$ is closed under direct limits. Using [13, Theorem 5.31] we conclude that Cogen $(T)$ is a covering class. 
Since every module has an injective envelope over an arbitrary ring, application of dual techniques to that applied in the silting case gives us the dual of Theorem 2.4. Moreover the dual of Corollary 2.12, i.e. cosilting classes are exactly the torsion-free classes which are covering, is valid for arbitrarily rings.

Theorem 3.5. Let $R$ be a ring and $E$ a fixed injective cogenerator for Mod-R. If $\mathcal{F}$ is a torsion-free class in Mod- $R$, the following are equivalent:

(1) $\mathcal{F}=\operatorname{Cogen}(T)$ for a cosilting module $T$;

(2) $\mathcal{F}$ is a covering class;

(3) there exists an $\mathcal{F}$-cover $\varepsilon: M \rightarrow E$;

(4) There exists an $\mathcal{F}$-precover $\varepsilon: M \rightarrow E$ such that $M \in \mathcal{F} \cap \mathcal{F}^{\perp}$.

Moreover, if $R$ is hereditary, then the above conditions are equivalent to:

(5) There exists an exact sequence $0 \rightarrow K \rightarrow M \rightarrow E \rightarrow V \rightarrow 0$ such that $M \in \mathcal{F}$, $V \in \mathcal{F}^{\circ}$ and $K \in \mathcal{F}^{\perp}$

In these conditions, if $K=\operatorname{Ker}(\varepsilon)$ then $M \oplus K$ is a cosilting module and $\mathcal{F}=$ Cogen $(M \oplus K)$.

Proof. The implication $(1) \Rightarrow(2)$ follows from Lemma 3.4 and $(2) \Rightarrow(3)$ is trivial. The implication $(3) \Rightarrow(4)$ follows from the dual of Lemma 3.3 , and $(4) \Rightarrow(1)$ is the dual of $(2) \Rightarrow(1)$ from Theorem 2.4 .

In the hereditary case the equivalence $(1) \Leftrightarrow(5)$ is the dual of the equivalence $(1) \Leftrightarrow(3)$ stated in Theorem 2.6.

Let us note that the equivalence $(1) \Leftrightarrow(2)$ was proved independently by Zhang and Wei, cf. [24, Theorem 3.5] and [25, Theorem 4.18].

In the following example we will see that the property $V \in \mathcal{F}^{\circ}$ cannot be deduced if $R$ is not hereditary.

Example 3.6. Let $R$ be the ring used in Example 2.8. If $(-)^{d}$ is the standard duality between right and left finitely presented modules, and $M$ is the silting module used in Example 2.8 then we can use the proof of [10, Corollary 3.7] to see that $M^{d}$ is a cosilting module and

$$
0 \longrightarrow S_{1}^{d} \longrightarrow P_{1}^{d} \oplus P_{1}^{d} \stackrel{{ }^{1} P_{1}^{d} \oplus \varphi^{d}}{\longrightarrow} R^{d} \longrightarrow U^{d} \longrightarrow 0
$$

is the exact sequence induced by the $\operatorname{Cogen}\left(M^{d}\right)$-cover $1_{P_{1}^{d}} \oplus \varphi^{d}$ for $R^{d}$ such that $\operatorname{Coker}\left(1_{P_{1}^{d}} \oplus \varphi^{d}\right)=U^{d}$ is not in $\operatorname{Cogen}\left(M^{d}\right)^{\circ}$.

We have also the dual of Proposition 2.13.

Proposition 3.7. Let $T$ be a cosilting module. If $\mathcal{F}=\operatorname{Cogen}(T)$ then for every $R$-module $X$ there exists a short exact sequence 


$$
0 \longrightarrow X \stackrel{v}{\longrightarrow} U \longrightarrow F \longrightarrow 0
$$

such that $v$ is a $\mathcal{F}^{\diamond}$-preenveloping for $X$ and $F \in \mathcal{F}$.

Corollary 3.8. Let $\mathcal{F}=\operatorname{Cogen}(T)$ for a cosilting module $T$. Then the class

$$
\mathcal{F}^{\perp}=\left\{X \in \operatorname{Mod}-R \mid \operatorname{Ext}_{R}^{1}(F, X)=0 \text { for all } F \in \mathcal{F}\right\}
$$

is an enveloping class.

Proof. Since $\mathcal{F}^{\diamond} \subseteq \mathcal{F}^{\perp}$, it follows that every $\mathcal{F}^{\diamond}$-preenvelope constructed in the previous proposition is a special $\mathcal{F}^{\perp}$-preenvelope. Therefore, it is enough to apply [13, Theorem 5.27] and [10, Corollary 4.8] to obtain the conclusion.

\section{References}

1. Adachi, T., Iyama, O. and Reiten, I., $\tau$-tilting theory, Compositio Mathematica 150 (2014), 415-452.

2. Anderson, F. W. and Fuller, K. R., Rings and Categories of Modules, 2. ed., Graduate Texts in Mathematics 13, Springer, New York, 1992.

3. Angeleri-Hügel, L. and Hrbek, M., Silting modules over commutative rings, Int. Math. Res. Not. 13 (2017), 4131-4151.

4. Angeleri-Hügel, L., Marks, F. and Vitória, J., Silting Modules, Int. Math. Res. Not. 2016 (2016), 1251-1284.

5. Angeleri-Hügel, L., Marks, F. and Vitória, J., Silting modules and ring epimorphisms, Adv. in Math. 303 (2016), 1044-1076.

6. Angeleri Hügel, L., Tonolo, A. and TrlifaJ, J., Tilting preenvelopes and cotilting precovers, Algebr. Represent. Theory 4 (2001), 155-170.

7. Auslander, M. and Reiten, I., Applications of contravariantly finite subcategories, Adv. in Math. 86 (1991), 111-152.

8. Bazzoni, S., Herzog, I., Př́inoda, P., Šaroch, J. and TrlifaJ, J., Pure projective tilting modules. preprint. arXiv: 1703.04745 [math.RT].

9. Breaz, S. and Modoi, G. C., Ideal cotorsion theories in triangulated categories. preprint. arXiv: 1501.06810 [math.CT].

10. Breaz, S. and Pop, F., Cosilting modules, Algebr. Represent. Theory. 20 (2017), 1305-1321.

11. Fu, X. H., Guil Asensio, P. A., Herzog, I. and Torrecillas, B., Ideal Approximation Theory, Adv. Math. 244 (2013), 750-790.

12. Fuchs, L., Infinite Abelian Groups. Vol. I, Pure and Applied Mathematics 36, Academic Press, New York-London, 1970.

13. Göвel, R. and TrlifaJ, J., Approximations and Endomorphism Algebras of Modules, 2nd ed., Walter de Gruyter, Berlin, 2012.

14. Hoshino, M., Kato, Y. and Miyachi, J.-I., On $t$-structures and torsion theories induced by compact objects, J. Pure Appl. Algebra 167 (2002), 15-35. 
15. Jasso, G., Reduction of $\tau$-tilting modules and torsion pairs, Int. Math. Res. Not. 16 (2014), 7190-7237.

16. Koenig, S. and YAng, D., Silting objects, simple-minded collections, t-structures and co-t-structures for finite-dimensional algebras, Doc. Math. 19 (2014), 403-438.

17. Mantese, F. and Tonolo, A., On the heart associated with a torsion pair, Topol. Appl. 159 (2012), 2483-2489.

18. MARKs, F. and ŠŤovíčEK, Torsion classes, wide subcategories and localisations, Bull. London Math. Soc. 49 (2017), 405-416.

19. Nicolas, P., Saorin, M. and Zvonareva, A., Silting Theory in triangulated categories with coproducts. preprint. arXiv: 1512.04700 [math.RT].

20. Parra Carlos, C. E. and Saorín, M., On hearts which are module categories, J. Math. Soc. Jpn. 68 (2016), 1421-1460.

21. Passman, D. S., A Course in Ring Theory, Texts and Readings in Mathematics 68, Hindustan Book Agency, New Delhi, 2013.

22. Psaroudakis, C. and Vitória, J., Realisation functors in tilting theory, Math. Z. 288 (2018), 965-1028.

23. Smalø, S. O., Torsion theory and tilting modules, Bull. Lond. Math. Soc. 16 (1984), $518-522$.

24. Zhang, P. and Wei, J., Quasi-cotilting modules and torsion-free classes, preprint. arXiv: 1601.01387 [math.RA].

25. Zhang, P. and Wei, J., Cosilting complexes and AIR-cotilting modules. J. Algebra 491 (2017), 1-31.

26. WEI, J., $\tau$-tilting theory and $\star$-modules, J. Algebra 414 (2014), 1-5.

Simion Breaz

Faculty of Mathematics and Computer Science

"Babeş-Bolyai" University

Str. Mihail Kogălniceanu 1, RO-400084

Cluj-Napoca

Romania

bodo@math.ubbcluj.ro

Received May 2, 2017

accepted August 9, 2017

in revised form July 28, 2017
Jan Žemlička

Department of Algebra, Faculty of Mathematics and Physics

Charles University in Prague

Sokolovská 83, CZ-186 75

Praha 8

Czech Republic

zemlicka@karlin.mff.cuni.cz 